MS092.003

Microsymposium

\title{
Encapsulation of diptheria anatoxin into ordered mesoporous silica
}

Marcia Carvalho De Abreu Fantini ${ }^{1}$, Tereza S. Martins ${ }^{2}$, Cristiano L. P. Oliveira ${ }^{1}$, Marcela A. Bordenalli ${ }^{3}$, Milene T. Franco ${ }^{3}$, Osvaldo A. Sant 'Anna ${ }^{3}$, Martin K. Rasmussen ${ }^{4}$, Heloisa N. Bordallo ${ }^{4}$

${ }^{1}$ Physics Institute-University Of Sao Paulo, Sao Paulo, Brazil, ${ }^{2}$ Instituto de Ciências Ambientais, Químicas e Farmacêuticas, Universidade Federal de São Paulo, São Paulo, Brazil, ${ }^{3}$ Instituto Butantan, São Paulo, Brazil, ${ }^{4}$ Niels Bohr Institute, Copenhagen University, Copenhagen, Denmark

E-mail: mfantini@if.usp.br

Different concentrations ( $w / w)$ of diphtheria anatoxin (D-ANA) were encapsulated into SBA-15 ordered mesoporous silica [1], which is composed by micropores (diameter less than $2 \mathrm{~nm}$ ), mesopores (mean diameter of $10 \mathrm{~nm}$ ) and macropores (diameter higher than $50 \mathrm{~nm}$ ). The encapsulation yield of the antigen diluted in phosphate-buffered saline (PBS) solution inside the silica, after a drying process, was determined by nitrogen adsorption isotherm (NAI), which provided BET surface area and the $\mathrm{BJH}$ pore size distribution. These results indicated that for a mass ratio of 5SBA-15:1ANA all mesopores are filled with the antigen, suggesting that it is the optimal concentration of D-ANA into SBA-15. Furthermore, the NAI results revealed that the micropores and mesopores are also filled by PBS. Analyzing the SAXS data, simulated by a theoretical model, which included form and structure factors [2], we concluded that D-ANA is inside the mesopores and that the mass ratio of 5SBA-15:1ANA is excessive, indicating the presence of D-ANA also in the macropore region. Transmission Electron Microscopy (TEM) images further confirmed these latter results. In-situ SAXS measurements of D-ANA release from the silica matrix were performed in mimetic intestine fluid. Dynamic Light Scattering (DLS) obtained from D-ANA in PBS showed that the antigen did not aggregate and hydrodynamic particle sizes between 5 to $10 \mathrm{~nm}$ were observed, which is also consistent with the ability of D-ANA to enter the mesopores. X-ray absorption spectroscopy with X-ray microscopy data at the carbon K-edge revealed that the D-ANA is distributed homogeneously in all the silica particles. The thermal stability of DANA increased when encapsulated inside SBA-15, as determined by Thermogravimetry (TG). The immunogenic complex DANA in the SBA-15 vehicle will be tested in mice in order to check the production of antibodies, regarding the exceptional adjuvant protecting characteristics of SBA-15 [3].

[1] Zhao D et al. (1998). Science 279(5350), 548-552.

[2] Sundblom A et al. (2010). J. Phys. Chem. C 113, 7706-13.

[3] Carvalho L V et al. (2010). Vaccine 28, 7829-36.

Keywords: diphtheria anatoxin, ordered mesoporous silica, SAXS 